\title{
Integrative analysis of gene expression associated with epilepsy in human epilepsy and animal models
}

\author{
HENGLING CHEN ${ }^{1}$, GUOZHENG XU ${ }^{2}$, HAO DU ${ }^{2}$, MINHAN YI ${ }^{3,4}$ and CHENHONG LI ${ }^{1}$ \\ ${ }^{1}$ The Laboratory of Membrane Ion Channels and Medicine, \\ Key Laboratory of Cognitive Science of State Ethnic Affairs Commission, \\ Hubei Key Laboratory of Medical Information Analysis and Tumor Diagnosis and Treatment, \\ College of Biomedical Engineering, South-Central University for Nationalities, Wuhan, Hubei 430074; \\ ${ }^{2}$ Department of Neurosurgery, Wuhan General Hospital of Guangzhou Military Command, Wuhan, Hubei 430070; \\ ${ }^{3}$ The State Key Laboratory of Medical Genetics, School of Life Sciences; ${ }^{4}$ Department of Medical Informatics, \\ Xiangya School of Medicine, Central South University, Changsha, Hunan 410078, P.R. China
}

Received May 11, 2015; Accepted March 21, 2016

DOI: $10.3892 / \mathrm{mmr} .2016 .5122$

\begin{abstract}
Epilepsy is a severe neuropsychiatric disorder, the cause of which remains to be elucidated. Genome-wide association studies, DNA microarrays and proteomes have been widely applied to identify the candidate genes involved in epileptogenesis, and integrative analyses are often capable of extracting more detailed biological information from the data. In the present study, a total number of 1,065 genes in different animal models were collected to construct an epilepsy candidate gene database. Further analyses suggested that the response to organic substances, the intracellular signaling cascade and neurological system processes were significantly enriched biological processes, and the mitogen-activated protein kinase pathway was identified as a putative epileptogenic signaling pathway. In addition, the five key genes, growth factor receptor bound 2 , amyloid $\beta$ (A4) precursor protein, transforming growth factor- $\beta$, vascular endothelial growth factor and cyclin-dependent kinase
\end{abstract}

Correspondence to: Professor Chenhong Li, The Laboratory of Membrane Ion Channels and Medicine, Key Laboratory of Cognitive Science of State Ethnic Affairs Commission, Hubei Key Laboratory of Medical Analysis and Tumor Diagnosis and Treatment, College of Biomedical Engineering, South-Central University for Nationalities, 182 Minyuan Road, Wuhan, Hubei 430074, P.R. China

E-mail: lichen@mail.scuec.edu.cn

Abbreviations: GWAS, genome-wide association studies; RT-qPCR, reverse transcription-quantitative polymerase chain reaction; GRB2, growth factor receptor bound 2; APP, amyloid $\beta$ (A4) precursor protein; TGF- $\beta$, transforming growth factor $\beta$; VEGF, vascular endothelial growth factor; CDKN1A, cyclin-dependent kinase inhibitor 1

Key words: epilepsy, animal models of seizures, differentially gene expression, pathway inhibitor 1, were identified as being critical as central nodes in the protein networks. Reverse transcription-quantitative polymerase chain reaction analysis revealed that these genes were all upregulated at the mRNA level in the epileptic loci compared with the resection margin of tissue samples from the same patients diagnosed with epilepsy. The data mining performed in the present study thus was shown to be a useful tool, which may contribute to obtaining further information on epileptic disorders and delineating the molecular mechanism of the associated genes.

\section{Introduction}

Epilepsy is one of the most common types of neurological disorder, which is caused by genetic and acquired factors (1). In terms of the genetics of idiopathic epilepsy, it is likely that an increasing number of genes encoding voltage-gated ion channel subunits, channel-associating proteins, neurotransmitters and neuropeptide receptors modify these phenomena in various types of idiopathic human epilepsy and in different animal models (2). Consistent with this hypothesis, it has been suggested that up to 1,000 genes may be involved in the pathogenesis and evolution of epilepsy (3). Identifying the mechanisms, with particular emphasis on candidate genes, is crucial to improve current understanding of epileptogenicity and epilepsy susceptibility.

The procedures of gene profiling by genomic, transcriptomic and proteomic analyses may provide a useful tool in identifying candidate genes, which are of importance in epilepsy. A number of genes have been revealed from genome-wide association studies (GWAS) for focal and generalized types of epilepsy (4). De novo mutations in GABA A receptor (GABR) 33 and UDP-N-acetylglucosamine transferase have previously been reported to be associated with epilepsy (5). Emerging evidence has indicated that synaptic transmission genes, including dynamin 1 , also cause epileptic encephalopathies (6). DNA microarrays and proteomes have been widely applied to analyze key molecular alterations in 
human and classical experimental epilepsy. According to previous reports, the differentially expressed genes represent several cellular events, including immune response (7), synaptic transmission (6,8), synaptic plasticity (9) and receptor processes (10-12). These genome-wide profiles have assisted in understanding the complex molecular mechanisms of epilepsy, however, integrative analyses are often capable of providing more detailed biological insight (13). The integration of bioinformatics offers promise in revealing the cellular networks of human epilepsy.

Lists of potential epileptogenesis-associated genes from GWAS, DNA microarrays and proteomes have become available and there is an increasing need to extract functional information from the high-throughput data. Thus, the present study aimed to comprehensively analyze the gene expression profile and search for potential key genes and pathways in epilepsy. As the different temporal-spatial gene expression patterns are directly related to the development of epileptic syndromes, the integrative analysis is crucial to providing novel insights into molecular events and supports the feasibility of a novel biomarker in epilepsy.

\section{Materials and methods}

Data sources. All the human, rat and mouse data obtained in the present study were from previously published results (http://devicelinked.com/ch/main2.html). The genes selected in the present study were those identified as significantly upregulated or downregulated between the epilepsy group and control group, which was determined as $\mathrm{P}<0.05$, compared with the control in the previous report. Gene ID conversions were performed on the collected data using Database to Database Conversions (bioDBnet; http://biodbnet.abcc.ncifcrf. gov/db/db2db.php) (14), which was then corrected and updated by The National Center for Biotechnology Information (http://www.ncbi.nlm.nih.gov/). The duplicate genes identified in the same organism were excluded in the final database. Venn diagrams (http://bioinfogp.cnb.csic.es/tools/venny/) were used for comparing the gene lists and identifying the associations between the collected sets.

Chromosome mapping and chromosome enrichment analysis. The chromosomal locus of each gene was determined using the Database to Database Conversions at bioDBnet. In addition, a search was performed on the Entrez Gene Database to correct the results. A hypergeometric test was used to determine whether the candidate genes for epilepsy were significantly enriched in specific chromosomes. The P-value of the test was defined as follows:

$$
p=1-\sum_{i=0}^{x-1}\left(\frac{K}{i}\right)\left(\frac{M}{N}\right)^{i}\left(1-\frac{M}{N}\right)^{k-i}
$$

In the above equation, $\mathrm{N}$ represents the number of all the genes annotated with chromosome location information, $\mathrm{K}$ represents the number of epilepsy candidate genes collected. $M$ represents the number of genes in a specific chromosome and $\mathrm{X}$ represents the number of candidate genes in the same chromosome. The present study determined the enrichment of the candidate genes in each chromosome for each species. $\mathrm{P}<0.05$, was considered to indicate a candidate gene, which was significantly enriched in a specific chromosome. The data source for the number of genes on each chromosome was the Ensembl genome browser release 68, July 2012 (http://uswest. ensembl.org/info/website/archives/assembly.html).

GO and KEGG pathway enrichment. The Database for Annotation, Visualization and Integrated Discovery website (DAVID; http://david.abcc.ncifcrf.gov/ $(15,16)$ was used to identify the significant GO and KEGG pathways in the differentially expressed gene lists. Multiple corrections were performed using Fisher's exact method, and DAVID provided a Benjamini-Hochberg false discovery rate-adjusted P-value.

Protein network analysis. The web-based expression analysis program, Cytoscape (http://www.cytoscape.org/), was used for network analysis, to describe the functional associations between genes or proteins using all human genes and the homologous genes from rat and mouse candidate gene lists.

$R T-q P C R$. Three pairs of brain tissue samples from 3 patients (Table I) with epilepsy were obtained from Wuhan General Hospital of Guangzhou Military Command (Wuhan, China), following approval of the Ethics Committee of South-Central University for Nationalities (Wuhan, China). Written informed consent was obtained from patients. On the day of RNA isolation, the tissues were disrupted and homogenzed using TRIzol reagent (Invitrogen; Thermo Fisher Scientific, Inc., Waltham, MA, USA) and used as a template for reserve transcription. A $25 \mu \mathrm{l}$ reaction was set up containing $1 \mu \mathrm{g}$ RNA, $0.5 \mu \mathrm{g}$ Oligo(dT), $5 \mu 1$ 5X M-MLV buffer, $0.5 \mathrm{mM}$ dNTP each, 25 units of RNasin Ribonuclease Inhibitor and 200 units M-MLV reverse transcriptase (all purchased from Promega, Madison, WI, USA). First-strand synthesis reaction was performed at $37^{\circ} \mathrm{C}$ for $1 \mathrm{~h}$. The qPCR primers used are presented in Table II, and the template samples used are listed in Table I. A total of $1 \mu \mathrm{l}$ cDNA was added to a $20 \mu \mathrm{l}$ reaction volume, resulting in a final concentration of $400 \mathrm{nM}$ forward primer, $400 \mathrm{nM}$ reverse primer and 1X SYBR Premix Ex Taq II (Takara Bio, Inc., Tokyo, Japan). The qPCR conditions were optimized in an Mx3005P system (Applied Biosystems; Thermo Fisher Scientific, Inc.). qPCR was performed at $95^{\circ} \mathrm{C}$ for $5 \mathrm{~min}$, followed by 40 cycles of $95^{\circ} \mathrm{C}$ for $30 \mathrm{sec}$ and $61^{\circ} \mathrm{C}$ for $1 \mathrm{~min}$. The relative expression values were normalized to the housekeeping gene, GAPDH, and calculated using the $\triangle \Delta \mathrm{Cq}$ method (17).

\section{Results}

Gene expression profiling and chromosome mapping. A total number of 1,065 genes were selected, which showed differential expression in the human epilepsy and animal models (Fig. 1A and B). The Candidate Epilepsy Gene Database (http://devicelinked.com/ch/main2.html) is a website built by the authors and was used to enable rapid querying of human, rat and mouse genes (Fig. 1C) and to download the candidate genes. The database presents gene ID, symbol and name, and provides the PubMed ID. The database is updated when new data is released. 
Table I. Sources of epilepsy samples used in reverse trancsription-quantitative polymerase chain reaction analysis.

\begin{tabular}{lccc}
\hline Patient & Age (years) & Gender & $\begin{array}{c}\text { Epileptic syndrome } \\
\text { diagnosis }\end{array}$ \\
\hline 1 & 29 & Male & Refractory epilepsy \\
2 & 18 & Male & Refractory epilepsy \\
3 & 37 & Male & Refractory epilepsy \\
\hline
\end{tabular}

Table II. Primer sequences for reveres transcription-quantitative PCR analysis.

\begin{tabular}{llc}
\hline Primer & \multicolumn{1}{c}{ Sequence (5'-3') } & Length (bp) \\
\hline GRB2s & AATGAAGCCGTCTTTTCCATT & 21 \\
GRB2a & ACGAGCTGAGCTTCAAAAGG & 20 \\
APPs & CCACAGAACATGGCAATCTG & 20 \\
APPa & TTTGGCACTGCTCCTGCT & 18 \\
TGF- $\beta 1 \mathrm{~s}$ & CTTCCAGCCGAGGTCCTT & 18 \\
TGF- $\beta 1 \mathrm{a}$ & CCCTGGACACCAACTATTGC & 20 \\
VEGFs & AGCTGCGCTGATAGACATCC & 20 \\
VEGFa & CTACCTCCACCATGCCAAGT & 20 \\
CDKN1As & CATGGGTTCTGACGGACAT & 19 \\
CDKN1Aa & AGTCAGTTCCTTGTGGAGCC & 20 \\
HPRT1s & GTTATGGCGACCCGCAG & 17 \\
HPRT1a & ACCCTTTCCAAATCCTCAGC & 20 \\
GAPDHs & AAGGTGAAGGTCGGAGTCAA & 20 \\
GAPDHa & AATGAAGGGGTCATTGATGG & 20 \\
\hline
\end{tabular}

PCR, polymerase chain reaction; GBR2, growth factor receptor bound 2; APP, amyloid $\beta$ (A4) precursor protein; TGF- $\beta$, transforming growth factor; VEGF, vascular endothelial growth factor; CDKN1A; cyclin-dependent kinase inhibitor 1; Hprt1, hyperparathyroidism 1; Gapdh, glyceraldehyde 3-phosphate dehydrogenase; a, antisense; s, sense.

To determine whether there was a chromosome preference for epilepsy, the differentially expressed genes were further mapped onto chromosomes. Based on chromosome locations, the epilepsy genes in humans were enriched on chromosome 1 (hypergeometric test, $\mathrm{P}=0.048)$, chromosome $5(\mathrm{P}=0.035)$ and chromosome $11(\mathrm{P}=0.045)$, as shown in Fig. 2A. The genes were enriched on chromosome $1(\mathrm{P}=0.001)$, chromosome 20 $(\mathrm{P}=0.004)$ and the mitochondrial chromosome $(\mathrm{P}=0.003)$ in the rat (Fig. 2B), and were predominantly located on chromosome $3(\mathrm{P}=0.008)$ and $17(\mathrm{P}=0.002)$ in the mouse (Fig. 2C).

Biological relevance and signaling pathways associated with epileptogenesis. The GO terms showing the highest level of significant enrichment are indicative of a bias in the type of functional classification. The statistically significant biological processes (BPs) with the highest levels of enrichment were response to organic substance, intracellular signaling cascade and neurological system process (adjusted $\mathrm{P}<0.01$ ). The cellular component ontology-term enrichment suggested that the important processes in the development of epileptogenesis occurred in the cytosol. The prevalent molecular functions were gated channel activity and structural constituent of ribosome (Table III). Using the KEGG database for analysis, it was found that the most significant pathways were the hsa04010 mitogen-activated protein kinase (MAPK) signaling pathway (31 counts; adjusted $\mathrm{P}=3.47 \mathrm{E}-07$ ) and hsa03010 Ribosome (18 counts; adjusted $\mathrm{P}=5.43 \mathrm{E}-07$ ). The rat and mouse data were similar to the human data obtained, and these can be downloaded from the website written by the authors (http://devicelinked.com/ch/main2.html).

Top networks during epileptogenesis. In addition to the classical pathways, the present study aimed to identify the genes, which were likely to be hubs. Growth factor receptor bound 2 (GRB2) was characterized as an interconnected node, suggesting it is associated with epileptogenesis (Fig. 3A). Amyloid $\beta$ (A4) precursor protein (APP) was also identified as a hub gene with a high level of connection (Fig. 3B). Transforming growth factor- $\beta$ (TGF- $\beta$ ) and vascular endothelial growth factor (VEGF) were key regulators of the underlying the protein network (Fig. 3C and D). Cyclin-dependent kinase inhibitor 1 (CDKN1A), also known as P21, may also exert its role as a central node (Fig. 3E). The networks also indicated that jun proto-oncogene, tumor necrosis factor, heat shock proteins and GABRA were also important genes (data not shown).

mRNA levels of GRB2, APP, TGF- $\beta, V E G F$ and $C D K N 1 A$ are upregulated in the cortex of patients with epilepsy. To confirm the hypothesis that the networks involving GRB2, APP, TGF- $\beta$, VEGF and CDKN1A may be important in human epileptic patients, the present study compared the mRNA levels of these genes between the epileptic locus, which was revealed using video-electroencephalography (EEG) recordings, and in the resection margin, where the incision was made in surgery, from the same surgically removed cortex sample from patients with drug-refractory epilepsy $(n=3)$. RT-qPCR analysis revealed that the mRNA levels of GRB2, APP, TGF- $\beta$, VEGF and CDKN1A were all upregulated in the epileptic cortex samples, compared with the control (resection margin) samples, in which GAPDH was used as a reference gene (Fig. 4). The housekeeping gene, hyperparathyroidism 1, exhibited identical values in the control and epileptic cortex samples.

\section{Discussion}

Several well-characterized models have been described previously, which represent the complex partial seizures observed in patients with epilepsy in several ways. Seizures can be induced by an excitotoxic compound for example kainic acid or pilocarpine, or by electric stimulation, referred to as kindling $(18,19)$. Animal models appear to be particularly informative for assessing the molecular mechanisms controlling the dynamic processes. Based on the analyses of the genomic, transcriptional and proteomic data from human patients and animal models in the present study, specific patterns regarding the candidate genes were observed, as follows: i) These genes show chromosomal preference, with a preference to chromosomes 1, 5 and 11 in humans. ii) Epilepsy-associated genes appear 


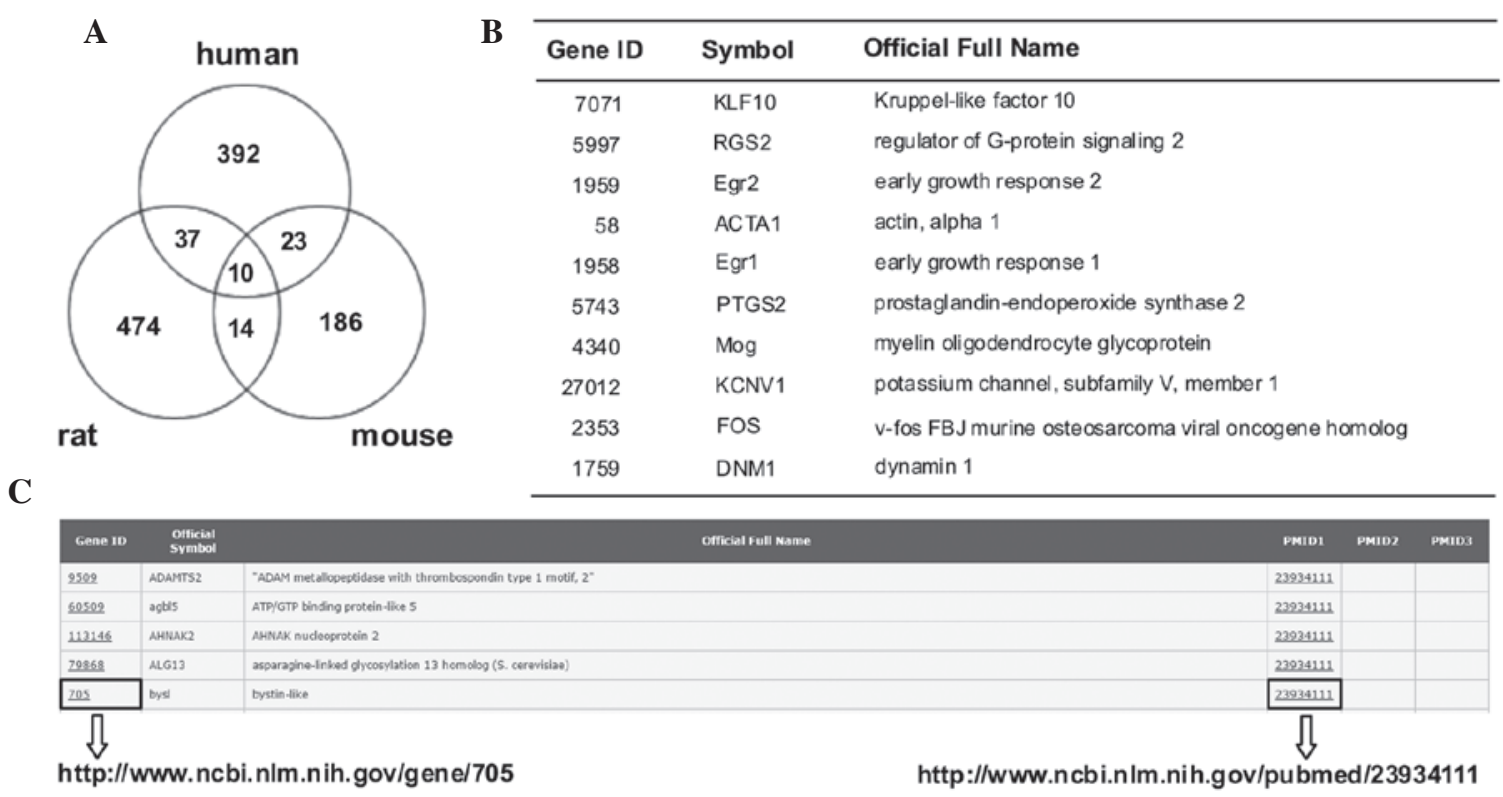

Figure 1. Comparison between candidate epilepsy genes. (A) Venn diagram indicating the number of genes differentially expressed in human epilepsy and animal models (rat and mouse) of epilepsy. (B) A total of 10 genes, were identified as being differentially expressed in humans and animal models. (C) Output of the Candidate Epilepsy Gene Database search.
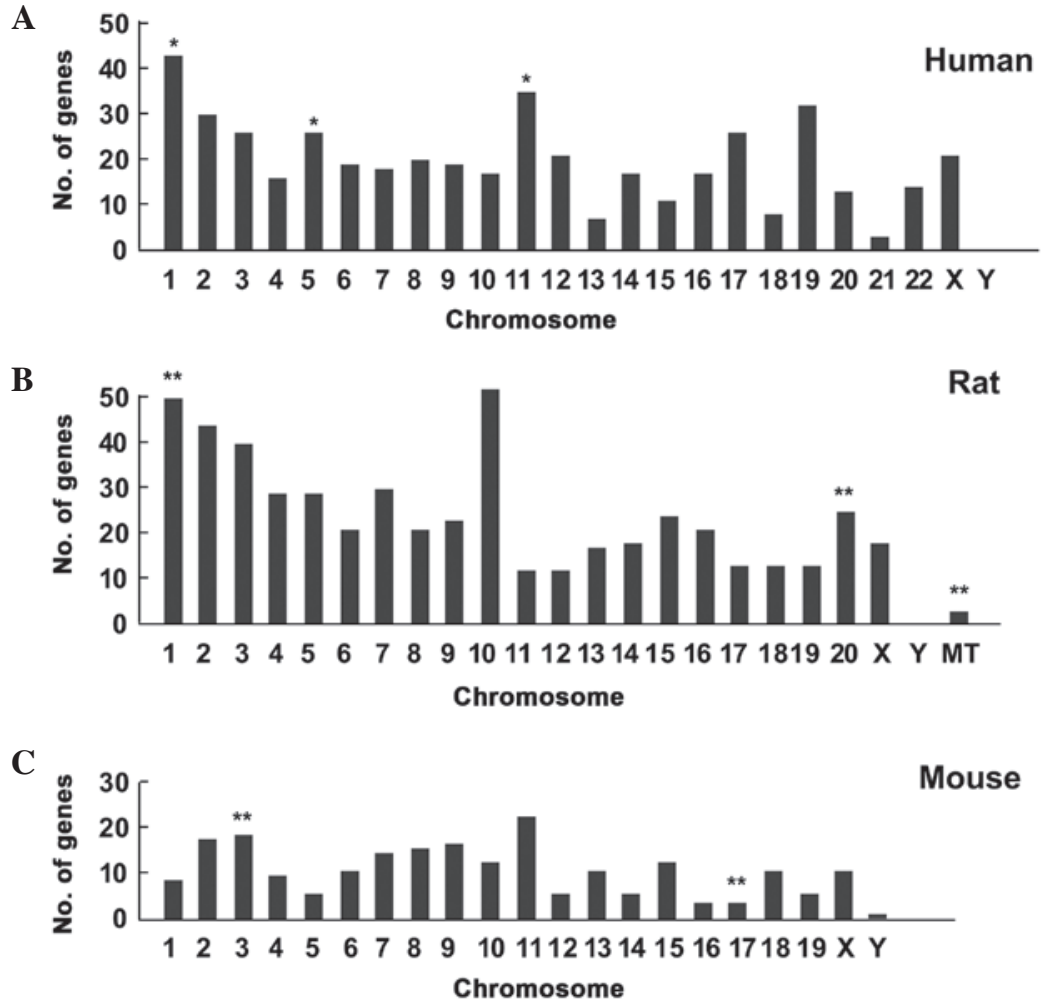

Figure 2. Distributions of candidate genes in chromosomes. The distribution of candidate genes in the (A) human, (B) rat and (C) mouse chromosomes were determined. The chromosome mapping information was based on Database to Database Conversions (bioDBnet; http://biodbnet.abcc.ncifcrf.gov/db/db2db. php). A hypergeometric test was performed. ${ }^{* *} \mathrm{P}<0.01$ and ${ }^{*} \mathrm{P}<0.05$ significantly enriched in the sample of genes associated with epilepsy (hypergeometric test). MT, mitochondrial chromosome.

to converge on specific BPs, including response to organic substance, intracellular signaling cascade and neurological system process. In addition, the MAPK signaling pathway is involved in multiple aspects of epileptic seizures. iii) Protein network analysis suggested that GRB2, APP, TGF- $\beta$, VEGF and
CDKN1A are key molecules involved in epileptic seizures, and the expression levels of these genes are upregulated in epilepsy loci, compared with controls in the same patient.

Considering the conserved synteny between the human, rat and mouse genomes, human chromosome 1 has been shown 
Table III. GO analysis of epilepsy candidate genes in humans.

\section{A, GOTERM_BP_FAT}

\begin{tabular}{lcccc}
\hline Term & Count & $\%$ & P-value & Adjusted P-value \\
\hline GO:0010033 response to organic substance & 60 & 13.0719 & $1.60 \mathrm{E}-13$ & $1.99 \mathrm{E}-10$ \\
GO:0007242 intracellular signaling cascade & 60 & 13.0719 & $8.54 \mathrm{E}-05$ & $3.71 \mathrm{E}-03$ \\
GO:0050877 neurological system process & 57 & 12.4183 & $1.95 \mathrm{E}-04$ & $6.88 \mathrm{E}-03$ \\
GO:0007267 cell-cell signaling & 50 & 10.8932 & $2.52 \mathrm{E}-11$ & $1.25 \mathrm{E}-08$ \\
GO:0042981 regulation of apoptosis & 48 & 10.4575 & $2.14 \mathrm{E}-06$ & $1.66 \mathrm{E}-04$ \\
GO:0043067 regulation of programmed cell death & 48 & 10.4575 & $2.79 \mathrm{E}-06$ & $2.09 \mathrm{E}-04$ \\
GO:0042592 homeostatic process & 48 & 10.4575 & $3.08 \mathrm{E}-06$ & $2.12 \mathrm{E}-04$ \\
GO:0019226 transmission of nerve impulse & 45 & 9.8039 & $4.37 \mathrm{E}-06$ & $2.71 \mathrm{E}-04$ \\
GO:0009719 response to endogenous stimulus & 43 & 9.3681 & $2.18 \mathrm{E}-15$ & $5.51 \mathrm{E}-12$ \\
GO:0007268 synaptic transmission & 36 & 7.8431 & $5.05 \mathrm{E}-09$ & $1.79 \mathrm{E}-06$ \\
\hline
\end{tabular}

\section{B, GOTERM_CC_FAT}

\begin{tabular}{|c|c|c|c|c|}
\hline Term & Count & $\%$ & P-value & Adjusted P-value \\
\hline GO:0005829 cytosol & 77 & 16.7755 & $1.51 \mathrm{E}-10$ & $1.76 \mathrm{E}-08$ \\
\hline GO:0044421 extracellular region part & 49 & 10.6753 & $2.44 \mathrm{E}-05$ & 4.72E-04 \\
\hline GO:0031982 vesicle & 42 & 9.1503 & $8.05 \mathrm{E}-07$ & $2.81 \mathrm{E}-05$ \\
\hline GO:0031988 membrane-bounded vesicle & 40 & 8.7145 & $8.12 \mathrm{E}-08$ & $4.05 \mathrm{E}-06$ \\
\hline GO:0031410 cytoplasmic vesicle & 40 & 8.7145 & $1.84 \mathrm{E}-06$ & 4.94E-05 \\
\hline GO:0005615 extracellular space & 40 & 8.7145 & 8.69E-06 & $1.90 \mathrm{E}-04$ \\
\hline GO:0042995 cell projection & 39 & 8.4967 & $2.98 \mathrm{E}-05$ & $5.20 \mathrm{E}-04$ \\
\hline GO:0016023 cytoplasmic membrane-bounded vesicle & 38 & 8.2788 & $3.01 \mathrm{E}-07$ & $1.17 \mathrm{E}-05$ \\
\hline GO:0043005 neuron projection & 33 & 7.1895 & $8.95 \mathrm{E}-10$ & 7.81E-08 \\
\hline GO:0045202 synapse & 30 & 6.5359 & $1.16 \mathrm{E}-07$ & $5.05 \mathrm{E}-06$ \\
\hline
\end{tabular}

\section{GOTERM_MF_FAT}

\begin{tabular}{lcccc}
\hline Term & Count & $\%$ & P-value & Adjusted P-value \\
\hline GO:0022836 gated channel activity & 23 & 5.0108 & $4.75 \mathrm{E}-05$ & $7.73 \mathrm{E}-03$ \\
GO:0003735 structural constituent of ribosome & 19 & 4.1394 & $8.50 \mathrm{E}-07$ & $5.56 \mathrm{E}-04$ \\
GO:0022843 voltage-gated cation channel activity & 17 & 3.7037 & $2.82 \mathrm{E}-06$ & $9.21 \mathrm{E}-04$ \\
GO:0022843 voltage-gated cation channel activity & 17 & 3.7037 & $2.82 \mathrm{E}-06$ & $9.21 \mathrm{E}-04$ \\
GO:0005248 voltage-gated sodium channel activity & 6 & 1.3071 & $3.66 \mathrm{E}-05$ & $7.94 \mathrm{E}-03$ \\
\hline
\end{tabular}

GO, Gene Ontology.

to be similar to regions of rat chromosome 2 and mouse chromosome 3 (20), on which the epilepsy candidate genes are all significantly enriched, suggesting that the orthologous segments on human chromosome 1 may be important in epileptogenicity. The associated genes also exhibit preference to human chromosome 5 and its orthologous segment rat chromosome 2 . The gene-rich human chromosome 11 is syntenic with a region of rat chromosome 1, on which the differentially expressed genes across rat models are predominantly located. Taken together, the conserved syntenic clusters of candidate genes on human chromosomes 1, 5 and 11 are likely to be essential for epileptogenesis.
The RT-qPCR analysis of human epilepsy tissue samples in the present study produced results consistent with those of previous studies. Specifically, GRB2 (21) and CDKN1A (22) have been reported to be upregulated in human epilepsy, and APP (23) and TGF- $\beta(24,25)$ were found to be upregulated following epileptic seizures in rat a model, based on DNA microarrays. Compared with wild-type mice, RT-qPCR has shown that the mRNA expression of VEGF is 1.44-fold higher in the hippocampus of VEGF Receptor-2 (Flk-1)-overexpressing mice, characterized by an elevated threshold for seizure induction (26). Immunostaining has also revealed that the protein levels of VEGF are increased 


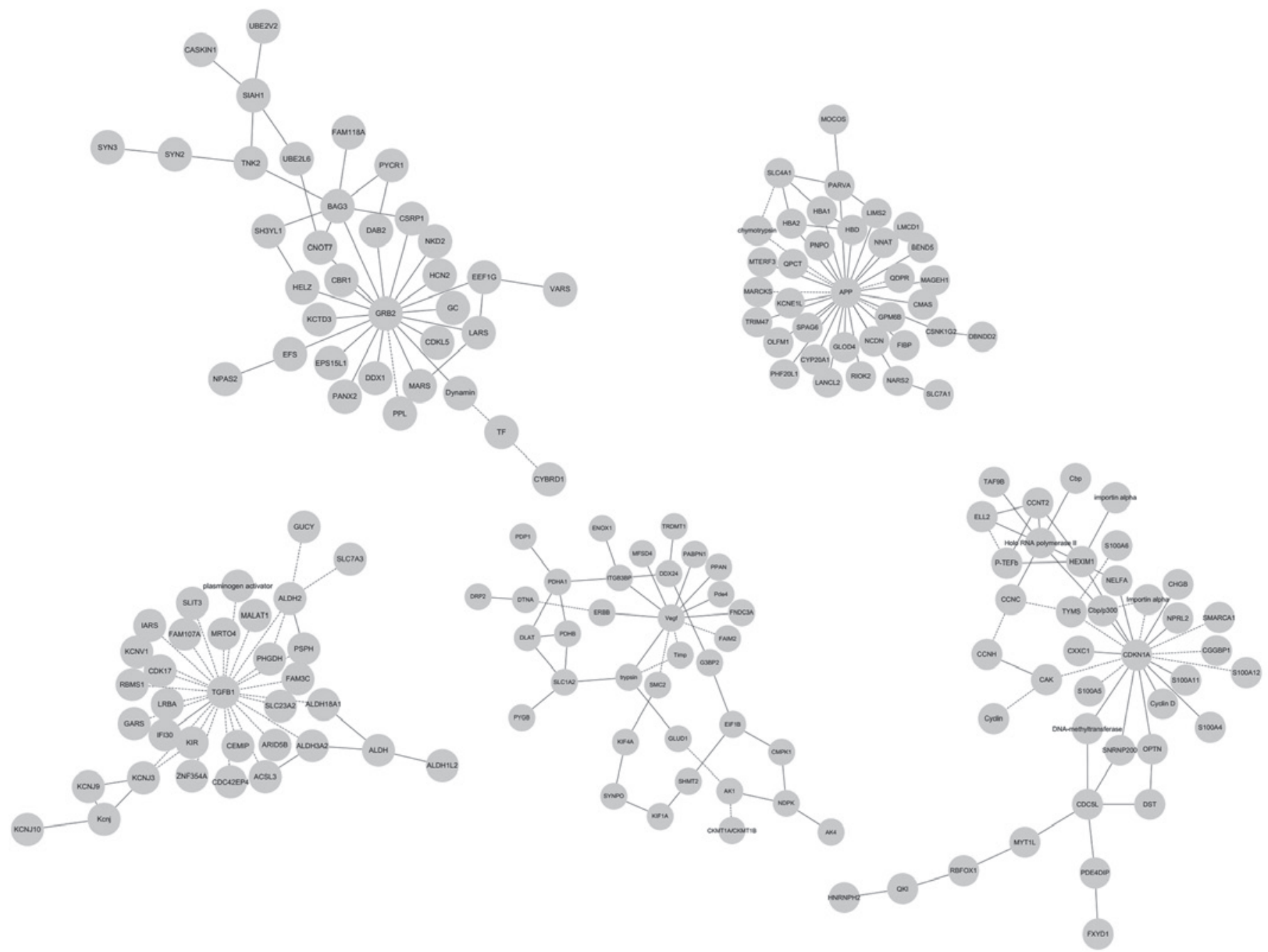

Figure 3. Integrated networks surrounding the hub genes. The networks surrounding the (A) GRB2, (B) APP, (C) TGF- $\beta$, (D) VEGF and (E) CDKN1A were determined. Data sets were analyzed using Cytoscape software. Genes are presented as nodes and the biological associations are presented as edges. Straight lines indicate direct interactions, and dashed lines indicate indirect interactions. GBR2, growth factor receptor bound 2; APP, amyloid $\beta$ (A4) precursor protein; TGF- $\beta$, transforming growth factor- $\beta$; VEGF, vascular endothelial growth factor; CDKN1A; cyclin-dependent kinase inhibitor 1.
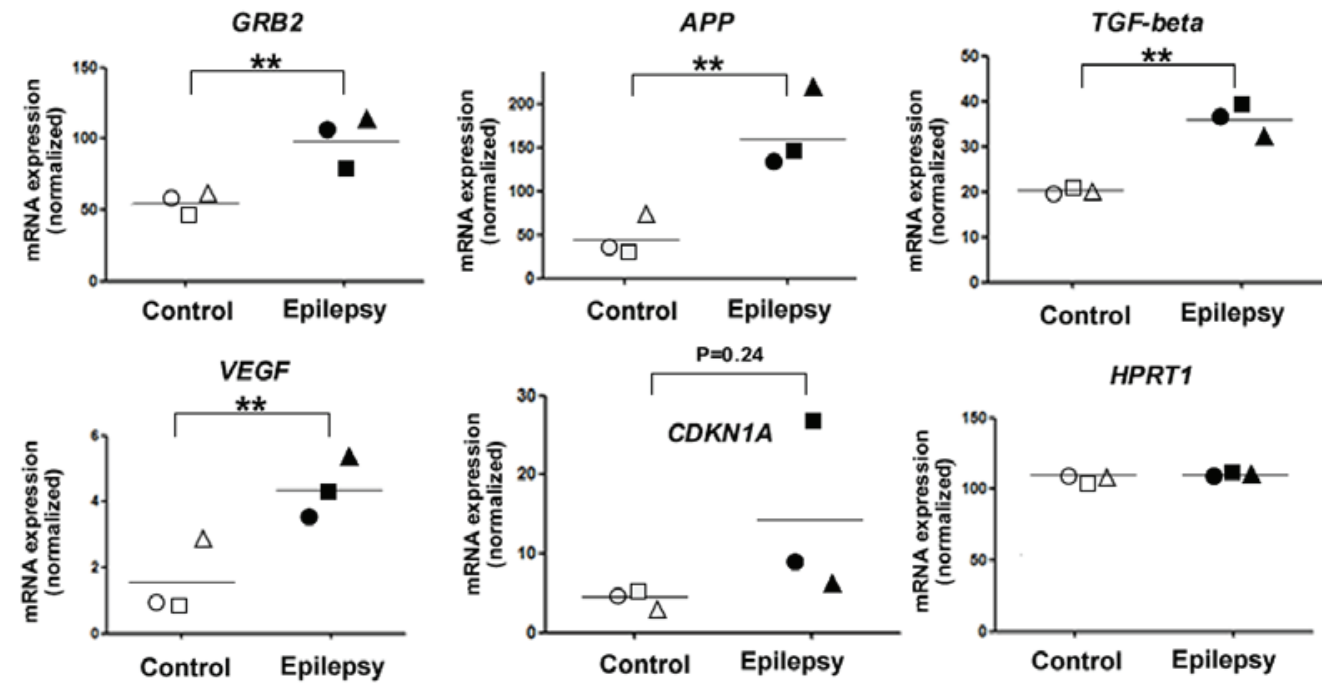

Figure 4. Fluorescent reverse-transcription-quantitative polymerase chain reaction analyses of the five key genes identified in patients with epilepsy. The Y-axis represents relative expression levels of the genes, the X-axis indicates the control and epilepsy tissue samples. The same shape indicates control and epilepsy samples from the same patient. All samples were measured in triplicate and data are presented as the mean expression ratio, ${ }^{* *} \mathrm{P}<0.05$. GBR2, growth factor receptor bound 2; APP, amyloid $\beta$ (A4) precursor protein; TGF- $\beta$, transforming growth factor- $\beta$; VEGF, vascular endothelial growth factor; CDKN1A; cyclin-dependent kinase inhibitor 1; HPRT1, hyperparathyroidism 1. 
in neurons and glia following pilocarpine-induced status epilepticus in rats (20).

In particular, the high proportion of genes from the GRB2 network may contribute to epileptogenesis or the recovery process $(27,28)$. The mutations in APP and its associated genes may lead to toxic accumulation of $A \beta$ protein fragments, which are important in temporal lobe epilepsy (29). The role of VEGF following seizures may be either protective or destructive (20). VEGF is important in initiating changes in the blood-brain barrier (BBB) by vascular remodeling, and BBB permeability in turn contributes to epileptogenesis (30). However, VEGF has also been shown to potentially protect vulnerable cells from the damage associated with seizures (20). Consistent with previous reports describing the role of the TGF- $\beta$ pathway in the development of neocortical epileptogenesis, TGF- $\beta$ may initiate and maintain cellular alterations as a putative signaling cascade $(31,32)$. Until now, there has been no report on the exact role of CDKN1A in epilepsy. A subset of these genes may serve as novel biomarkers to improve the current diagnosis of epilepsy. The large-scale expression profiling performed in the present study may provide clues on the epigenetic mechanisms through the key modules, described above.

The results of the present study offer insights into the pathogenesis of epilepsy, and a number of the candidate genes detected may be important in epilepsy. However, further investigations are required to determine which genes initiate the occurrence of epilepsy and to determine the exact roles.

\section{Acknowledgements}

This study was supported by the National Natural Science foundation of China (grant nos. 30972848, 31500996 and 81271234) and the Special Funds of Basic research operating expenses for universities of China (grant nos. CZY14016, CZZ11009, CZW14022 and CZW14058).

\section{References}

1. Berg AT, Berkovic SF, Brodie MJ, Buchhalter J, Cross JH, van Emde Boas W, Engel J, French J, Glauser TA, Mathern GW, et al: Revised terminology and concepts for organization of seizures and epilepsies: Report of the ILAE commission on classification and terminology, 2005-2009. Epilepsia 51: 676-685, 2010.

2. Meldrum BS and Rogawski MA: Molecular targets for antiepileptic drug development. Neurotherapeutics 4: 18-61, 2007.

3. Frankel WN: Detecting genes in new and old mouse models for epilepsy: A prospectus through the magnifying glass. Epilepsy Res 36: 97-110, 1999.

4. Jensen FE: Epilepsy in 2013: Progress across the spectrum of epilepsy research. Nat Rev Neurol 10: 63-64, 2014.

5. Epi4K Consortium; Epilepsy Phenome/Genome Project, Allen AS, Berkovic SF, Cossette P, Delanty N, Dlugos D, Eichler EE, Epstein MP, Glauser T, et al: De novo mutations in epileptic encephalopathies. Nature 501: 217-221, 2013.

6. EuroEPINOMICS-RES Consortium, Epilepsy Phenome/Genome Project, Epi4K Consortium: De novo mutations in synaptic transmission genes including DNM1 cause epileptic encephalopathies. Am J Hum Genet 95: 360-370, 2014.

7. Vezzani A, French J, Bartfai T and Baram TZ: The role of inflammation in epilepsy. Nat Rev Neurol 7: 31-40, 2011.

8. Fukata Y, Lovero KL, Iwanaga T, Watanabe A, Yokoi N, Tabuchi K, Shigemoto R, Nicoll RA and Fukata M: Disruption of LGI1-linked synaptic complex causes abnormal synaptic transmission and epilepsy. Proc Natl Acad Sci 107: 3799-3804, 2010.

9. Vissel B, Royle G, Christie B, Schiffer HH, Ghetti A, Tritto T, Perez-Otano I, Radcliffe RA, Seamans J, Sejnowski T, et al: The role of RNA editing of kainate receptors in synaptic plasticity and seizures. Neuron 29: 217-227, 2001
10. Gorter JA, van Vliet EA, Aronica E, Breit T, Rauwerda H, Lopes da Silva FH and Wadman WJ: Potential new antiepileptogenic targets indicated by microarray analysis in a rat model for temporal lobe epilepsy. J Neurosci 26: 11083-11110, 2006.

11. Tecott LH, Sun LM, Akana SF, Strack AM, Lowenstein DH, Dallman MF and Julius D: Eating disorder and epilepsy in mice lacking 5-HT2c serotonin receptors. Nature 374: 542-546, 1995.

12. Brooks-Kayal AR, Shumate MD, Jin H, Rikhter TY and Coulter DA: Selective changes in single cell GABAA receptor subunit expression and function in temporal lobe epilepsy. Nat Med 4: 1166-1172, 1998.

13. Lukasiuk K and Pitkänen A: Gene and protein expression in experimental status epilepticus. Epilepsia 48 (Suppl 8): S28-S32, 2007.

14. Mudunuri U, Che A, Yi M and Stephens RM: bioDBnet: The biological database network. Bioinformatics 25: 555-556, 2009.

15. Huang da W, Sherman BT and Lempicki RA: Systematic and integrative analysis of large gene lists using DAVID bioinformatics resources. Nat Protoc 4: 44-57, 2009.

16. Huang da W, Sherman BT and Lempicki RA: Bioinformatics enrichment tools: Paths toward the comprehensive functional analysis of large gene lists. Nucleic Acids Res 37: 1-13, 2009.

17. Livak KJ and Schmittgen TD: Analysis of relative gene expression data using real-time quantitative PCR and the $2^{-\Delta \Delta C}$ method. Methods 25: 402-408, 2001.

18. Sutula TP: Secondary epileptogenesis, kindling and intractable epilepsy: A reappraisal from the perspective of neural plasticity. Int Rev Neurobiol 45: 355-386, 2001.

19. Löscher W: Critical review of current animal models of seizures and epilepsy used in the discovery and development of new antiepileptic drugs. Seizure 20: 359-368, 2011.

20. Gibbs RA, Weinstock GM, Metzker ML, Muzny DM, Sodergren EJ, Scherer S, Scott G, Steffen D, Worley KC, Burch PE, et al: Genome sequence of the Brown Norway rat yields insights into mammalian evolution. Nature 428: 493-521, 2004

21. Arion D, Sabatini M, Unger T, Pastor J, Alonso-Nanclares L, Ballesteros-Yáñez I, García Sola R, Muñoz A, Mirnics K and DeFelipe J: Correlation of transcriptome profile with electrical activity in temporal lobe epilepsy. Neurobiol Dis 22: 374-387, 2006.

22. Beaumont TL, Yao B, Shah A, Kapatos G and Loeb JA: Layer-specific CREB target gene induction in human neocortical epilepsy. J Neurosci 32: 14389-14401, 2012.

23. Winden KD, Karsten SL, Bragin A, Kudo LC, Gehman L, Ruidera J, Geschwind DH and Engel J Jr: A systems level, functional genomics analysis of chronic epilepsy. PloS One 6: e20763, 2011.

24. Okamoto OK, Janjoppi L, Bonone FM, Pansani AP, da Silva AV, Scorza FA and Cavalheiro EA: Whole transcriptome analysis of the hippocampus: Toward a molecular portrait of epileptogenesis. BMC Genomics 11: 230, 2010.

25. Christensen KV, Leffers H, Watson WP, Sánchez C, Kallunki P and Egebjerg J: Levetiracetam attenuates hippocampal expression of synaptic plasticity-related immediate early and late response genes in amygdala-kindled rats. BMC Neurosci 11: 9, 2010.

26. Nikitidou L, Kanter-Schlifke I, Dhondt J, Carmeliet P, Lambrechts D and Kokaia M: VEGF receptor-2 (Flk-1) overexpression in mice counteracts focal epileptic seizures. PloS One 7: e40535, 2012.

27. Pitkänen A and Lukasiuk K: Molecular and cellular basis of epileptogenesis in symptomatic epilepsy. Epilepsy Behav 14 (Suppl 1): S16-S25, 2009.

28. Newton SS, Collier EF, Bennett AH, Russell DS and Duman RS: Regulation of growth factor receptor bound 2 by electroconvulsive seizure. Brain Res 129: 185-188, 2004.

29. Noebels J: A perfect storm: Converging paths of epilepsy and Alzheimer's dementia intersect in the hippocampal formation. Epilepsia 52 (Suppl 1): S39-S46, 2011

30. Morin-Brureau M, Lebrun A, Rousset MC, Fagni L, Bockaert J, de Bock F and Lerner-Natoli M: Epileptiform activity induces vascular remodeling and zonula occludens 1 downregulation in organotypic hippocampal cultures: Role of VEGF signaling pathways. J Neurosci 31: 10677-10688, 2011.

31. Friedman A, Kaufer D and Heinemann U: Blood-brain barrier breakdown-inducing astrocytic transformation: Novel targets for the prevention of epilepsy. Epilepsy Res 85: 142-149, 2009.

32. Friedman A: Blood-brain barrier dysfunction, status epilepticus, seizures and epilepsy: A puzzle of a chicken and egg? Epilepsia 52 (Suppl 8): S19-S20, 2011. 